\title{
Clinical Outcomes of Arthroscopic Rotator Cuff Repair Using Poly Lactic-co-glycolic Acid Plus $\beta$-tricalcium Phosphate Biocomposite Suture Anchors
}

\author{
Seok Won Chung ${ }^{\bowtie}$, Kyung-Soo Oh, Sung Jin Kang, Jong Pil Yoon ${ }^{1}$, Joon Yub Kim² \\ Department of Orthopaedic Surgery, Konkuk University School of Medicine, Seoul, ${ }^{1}$ Department of Orthopaedic Surgery, Kyungpook University College of \\ Medicine, Daegu, ${ }^{2}$ Department of Orthopaedic Surgery, Myongji Hospital, Goyang, Korea
}

\begin{abstract}
Background: This study is performed to evaluate anchor-related outcomes and complications after arthroscopic rotator cuff repair using $30 \% \beta$-tricalcium phosphate ( $\beta$-TCP) with $70 \%$ poly lactic-Co-glycolic acid (PLGA) biocomposite suture anchors.

Methods: A total of 78 patients (mean age, $61.3 \pm 6.9$ years) who underwent arthroscopic medium-to-large full-thickness rotator cuff tear repair were enrolled. The technique employed 30\% $\beta$-TCP with 70\% PLGA biocomposite suture anchors at the medial row (38 patients, Healix $\mathrm{BR}^{\mathrm{TM}}$ anchor [Healix group]; 40 patients, Fixone anchor B [Fixone group]). The radiologic outcomes (including perianchor cyst formation or bone substitution) and anatomical outcomes of the healing failure rate were evaluated using magnetic resonance imaging at least 6 months after surgery, the pain visual analogue scale at 3, 6 months, and final follow-up visit, and American Shoulder and Elbow Surgeons scores at least 1 year postoperatively. Anchor-related complications were also evaluated.

Results: The perianchor cyst formation incidence was similar for both groups (60.5\%, Healix group; $60.0 \%$, Fixone group; $p=0.967)$, although severe perianchor cyst incidence was slightly lower in the Fixone group (15.0\%) than in the Healix group (21.1\%). There was no occurrence of anchor absorption and bone substitution. No differences were observed in the healing failure rate $(13.2 \%$, Healix group; $15.0 \%$, Fixone group; $p=0.815$ ) and functional outcome between groups (all $p>0.05)$. Anchor breakage occurred in 5 patients ( 2 Healix anchors and 3 Fixone anchors); however, there were no major anchor-related complications in either group.

Conclusions: No differences were observed in the clinical outcomes of the Healix and Fixone groups, neither were there any accompanying major anchor-related complications.
\end{abstract}

(Clin Shoulder Elbow 2018;21(1):22-29)

Key Words: Biocomposite suture anchor; Fixone; Healix; Rotator cuff repair; Outcomes

\section{Introduction}

Rotator cuff tear is a common injury that causes pain and functional disability. With aging of the population and growing participation in sports activities, the incidence of rotator cuff tear has increased rapidly. ${ }^{1)}$ Consequently, arthroscopic rotator cuff repair, a widely accepted treatment for full-thickness rotator cuff tears, is frequently being performed. The use of suture anchors is an integral part of arthroscopic rotator cuff repair, to provide fixation of the rotator cuff tendon to the bone.

The initial suture anchors were made from metallic materials; however, complications associated with the use of metallic anchors, such as loosening, migration, cartilage damage, difficulty with revision surgery, and interference with postoperative magnetic resonance imaging (MRI), ${ }^{2-4)}$ led to the development of bioabsorbable suture anchors as an alternative. The advantages

Received December 11, 2017. Revised January 16, 2018. Accepted February 7, 2018.

Correspondence to: Seok Won Chung

Department of Orthopaedic Surgery, Center for Shoulder and Elbow Surgery, Konkuk University School of Medicine, 120 Neungdong-ro, Gwangjin-gu, Seoul 05029, Korea

Tel: +82-2-2030-7604, Fax: +82-2-2030-7748, E-mail: smilecsw@gmail.com

IRB approval: Konkuk University Hospital (No. KUH1060114).

Financial support: This research was supported by a grant from the Korea Health Technology R\&D Project through the Korea Health Industry Development Institute, funded by the Ministry of Health \& Welfare, Republic of Korea (grant number: HI15C1856). Conflict of interests: None. 
of bioabsorbable anchors include their ability of being absorbed over time, reduced complications related to migration, less artifacts in MRI, and degree of tendon-to-bone repair similar to that of metallic anchors. ${ }^{5}$ However, bioabsorbable anchors have also been reported to cause anchor-related complications such as synovitis, osteolysis, intraosseous cyst formation, systemic allergic response, and anchor breakage. ${ }^{6-9)}$ Polyglycolic acid (PGA) polymers were first used as bioabsorbable anchors, but the rapid degradation of PGA and loss of strength at a short-term postoperative period soon resulted in their discontinuation. ${ }^{10)}$ Thereafter, poly-L-lactic acid (PLLA) polymers, which have slower degradation periods, were used. However, another concern that has arisen is that an excessively long period of degradation would not allow complete osseous replacement, and may form intraosseous foreign bodies. ${ }^{11)}$

To control the degradation period of bioabsorbable anchors and to reinforce their mechanical properties, copolymers such as poly-D,L-lactide from L-lactide and D-lactide (PLDLA), and PLLA with PGA, have been developed. ${ }^{12)}$ Further, biocomposite materials which blend with tricalcium phosphate (TCP), have also been manufactured to facilitate bone formation. These are expected to yield minimal tissue reaction, good bone ingrowth, and sufficient initial fixation strength and durability during the healing for rotator cuff repair. ${ }^{13)}$

Considering a biocomposite suture anchor comprising 30\% $\beta$-TCP and 70\% poly-lactic-co-glycolic acid (PLGA), the Healix $\mathrm{BR}^{\mathrm{TM}}$ anchor (DePuy Mitek, Raynham, MA, USA) was developed. Several studies showed good results of the Healix $B^{T M}$ anchor, especially in terms of biodegradation and perianchor cyst formation. ${ }^{14-16)}$ Barber et al. ${ }^{14)}$ showed the Healix $\mathrm{BR}^{\mathrm{TM}}$ anchor was completely degraded and no remnant was present 3 years after implantation. In addition, Kim et al. ${ }^{15)}$ reported that the Healix $\mathrm{BR}^{\mathrm{TM}}$ anchor decreased the severity of perianchor cyst formation after rotator cuff repair, and similarly Randelli et al. ${ }^{16)}$ reported the Healix $\mathrm{BR}^{\mathrm{TM}}$ anchor showed excellent biological efficacy, without causing significant cystic lesions at a mean of 29 months after Bankart repair.

Recently, a new biocomposite suture anchor for rotator cuff repair has been developed, comprising 30\% $\beta$-TCP and 70\% PLGA (Fixone anchor B; Aju Medical, Seoul, Korea); its biomechanical properties were reported to be satisfactory in both air and saline conditions. ${ }^{17)}$ However, no clinical study has so far investigated the outcomes of using the Fixone anchor B.

This study was therefore undertaken to evaluate the anchorrelated outcomes and complications after arthroscopic rotator cuff repair using the newly developed Fixone anchor B, and to compare them with procedures using the Healix $\mathrm{BR}^{\mathrm{TM}}$ anchor. Since both have the same integral components, we hypothesized that the Fixone anchor B would show as good clinical results as the Healix $\mathrm{BR}^{\mathrm{TM}}$ anchor.

\section{Methods}

\section{Patients}

Our study protocol was approved by the Institutional Review Board of Konkuk University Hospital (No. KUH1060114). All authors, their immediate family, and any research foundation with which they were affiliated did not receive any financial payments or other benefits from any commercial entity related to the subject of this article. This was a retrospective, comparative cohort study. Of the 338 patients who underwent arthroscopic repair for full-thickness rotator cuff tears at the authors' hospital between December 2013 and September 2016, we included patients who underwent the repair surgery using biocomposite suture anchors comprising PLGA and $\beta$-TCP components at the medial row for medium-to-large-sized tears, had postoperative MRI scans at least 6 months after surgery, and various functional outcome measurements at least 1 year after surgery. The exclusion criteria for patients were: use of other types of suture anchors at the medial row $(n=121)$; presence of partial-thickness rotator cuff tears $(n=23)$, small tears $(n=21)$, massive tears $(n=42)$, and isolated subscapularis tears $(n=5)$; prior surgery on the shoulder being studied ( $n=6)$; and loss to follow-up within 1 year after surgery $(n=42)$. Partial, small, and massive tears were excluded since their repair methods were not uniformly suture bridge techniques, and we believe that the characteristics and course of recovery of massive tears differ from those of mediumto-large tears. ${ }^{18)}$

Finally, 78 patients (40 men, 38 women) with an average age of $61.3 \pm 6.9$ years (range, $45-75$ years) were enrolled. The patients who fulfilled the inclusion criteria were divided into two series, based on the period in which they were treated. The first series consisted of patients who underwent surgery between December 2013 and November $2015(\mathrm{n}=38)$, and the second series were treated between December 2015 and September $2016(n=40)$. For the first 38 consecutive patients, we used the

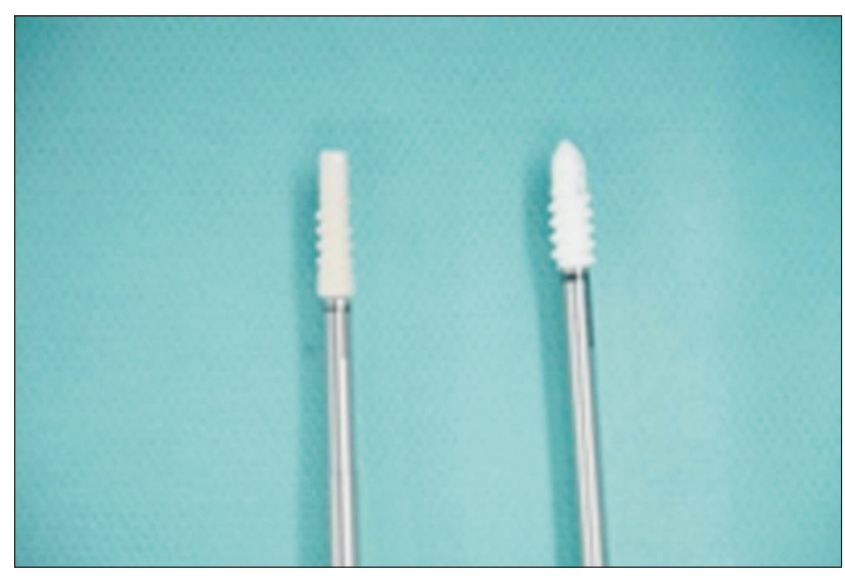

Fig. 1. Healix $\mathrm{BR}^{\mathrm{TM}}$ anchor (left; DePuy Mitek, Raynham, MA, USA) and Fixone anchor B (right; Aju Medical, Seoul, Korea). 
Healix BR ${ }^{T M}$ Anchor (PLGA $\beta$-TCP biocomposite; Healix group) for the medial row fixation, and the Fixone anchor B (PLGA $/ \beta$ TCP biocomposite; Fixone group) for medial row fixation was used for the next 40 consecutive patients (Fig. 1). The replacement using the Fixone anchor B was considered from December 2015 due to the increasing clinical and academic interests in this newly developed biocomposite anchor, and to assess whether the clinical outcomes of the in-country developed Fixone anchor $\mathrm{B}$ was as successful as the previously used Healix $\mathrm{BR}^{\mathrm{TM}}$ anchor having the same material composition. All patients underwent arthroscopic subacromial decompression with acromioplasty and complete rotator cuff repair using the suture bridge technique. The average age at the time of surgery was $61.6 \pm 8.0$ years in the Healix group, and $61.1 \pm 5.8$ years in the Fixone group; the group comprised 20 men and 18 women in the Healix group, and 20 men and 20 women in the Fixone group. The mean follow-up period was $14.9 \pm 6.4$ months for the Healix group, and $13.2 \pm 2.1$ months for the Fixone group.

\section{Demographic and Clinical Data}

Various demographic and clinical factors that affect the biocomposite anchor performance and clinical outcomes after surgery include age, sex, symptom duration, bone mineral density, traumatic onset, steroid injection history, tear size, fatty infiltration of each rotator cuff muscle, number of anchors used, and concomitant biceps procedures; these factors were prospectively collected and evaluated. The bone mineral density was measured at the last outpatient visit before surgery, using dual energy X-ray absorptiometry, and the lowest T-score of the proximal femur and lumbar spine, except for the value for the Ward's area of the proximal femur, was recorded..$^{19)}$ The tear size was measured arthroscopically using a calibrated probe at the time of surgery, and classified in accordance with the rating system of DeOrio and Cofield. ${ }^{20)}$ The fatty infiltration of each rotator cuff muscle (supraspinatus, infraspinatus, and subscapularis) was evaluated using the preoperative MRI findings, in accordance with the criteria established by Goutallier et al. ${ }^{211}$ and modified by Fuchs et al. ${ }^{22)}$ The demographic and clinical data of the two groups are summarized in Table 1, and there were no significant

Table 1. Comparison of the Demographic and Clinical Data between the Two Groups

\begin{tabular}{|c|c|c|c|}
\hline Variable & Healix group $(\mathrm{n}=38)$ & Fixone group $(n=40)$ & $p$-value \\
\hline \multicolumn{4}{|l|}{ Demographics and medical history } \\
\hline Age (yr) & $61.6 \pm 8.0$ & $61.1 \pm 5.8$ & 0.763 \\
\hline Sex (male:female) & 20:18 & 20:20 & 0.816 \\
\hline Symptom duration (mo) & $21.7 \pm 23.2$ & $24.9 \pm 21.1$ & 0.521 \\
\hline Bone mineral density (lowest T-score) & $-1.7 \pm 0.9$ & $-1.4 \pm 1.3$ & 0.451 \\
\hline Traumatic event (yes:no) & 14:24 & $11: 29$ & 0.377 \\
\hline History of past steroid injection (yes:no) & 10:28 & $16: 24$ & 0.200 \\
\hline \multicolumn{4}{|l|}{ Surgery related and anatomical variable } \\
\hline No. of medial anchors used (1:2:3) & $7: 28: 3$ & $4: 35: 1$ & 0.280 \\
\hline Treatment of LHBT (none:tenodesis:tenotomy) & $15: 11: 12$ & $14: 6: 20$ & 0.178 \\
\hline Tear classification (medium:large) & $22: 16$ & 24:16 & 0.835 \\
\hline Tear size of anteroposterior dimension $(\mathrm{cm})$ & $28.2 \pm 14.2$ & $24.7 \pm 11.2$ & 0.272 \\
\hline Amount of retraction $(\mathrm{cm})$ & $21.4 \pm 15.1$ & $20.9 \pm 13.7$ & 0.891 \\
\hline \multicolumn{4}{|l|}{ Fatty infiltration grade } \\
\hline Supraspinatus & $2.0 \pm 1.0$ & $2.0 \pm 1.1$ & 0.816 \\
\hline Infraspinatus & $1.8 \pm 1.2$ & $1.5 \pm 1.0$ & 0.279 \\
\hline Subscapularis & $1.3 \pm 1.1$ & $1.2 \pm 1.1$ & 0.637 \\
\hline \multicolumn{4}{|l|}{ Outcome related variable } \\
\hline Preoperative VAS for pain & $6.1 \pm 2.2$ & $6.3 \pm 2.0$ & 0.680 \\
\hline Preoperative ASES score & $56.4 \pm 15.7$ & $56.1 \pm 17.9$ & 0.930 \\
\hline
\end{tabular}

Values are presented as mean \pm standard deviation or number only. The Healix group includes patients who underwent arthroscopic rotator cuff repair using the Healix medial anchor (DePuy Mitek, Raynham, MA, USA), and the Fixone group includes patients who underwent the surgery using the Fixone medial anchor (Aju Medical, Seoul, Korea). Fatty infiltration is graded in accordance with the criteria by Goutallier et al. ${ }^{21}$

LHBT: long head of the biceps tendon, VAS: visual analogue scale, ASES: American Shoulder and Elbow Surgeons. 
differences in every variable between both groups.

\section{Surgical Procedures and Rehabilitation}

All surgical procedures were performed by a single surgeon at a single institute, using a uniform surgical technique. Subacromial decompression and acromioplasty were performed to create a flat acromion in all patients. Biceps tenotomy $(\mathrm{n}=32)$ or tenodesis $(n=17)$ was performed on patients with dislocation, subluxation, and tears involving more than $50 \%$ of the long head of the biceps tendon or in cases of a symptomatic degenerative superior labral anterior and posterior lesion, depending on the age or activity level of the patients. No patient underwent distal clavicular resection or coracoplasty. The margin of the tear was debrided to obtain better-quality tendon tissues. For reattachment of the rotator cuff tendons, a cancellous bone bed was prepared using a bur, until bleeding occurred. Procedures to mobilize the tendon were performed if the mobility of the tendon was insufficient for repair. All patients underwent arthroscopic suture bridge repair, and all knots were tied securely by applying a self-locking, sliding knot. For the first 38 consecutive patients, a 4.5-mm Healix $\mathrm{BR}^{\mathrm{TM}}$ anchor was used for the medial row fixation, and for the next 40 consecutive patients, a 5.5-mm Fixone anchor $\mathrm{B}$ was used for the medial row fixation. For lateral row fixation, several lateral row fixation anchors, such as SwiveLock ${ }^{\circledR}$ C anchor (Arthrex Inc., Naples, FL, USA), Footprint anchor (Smith \& Nephew, Memphis, TN, USA), Fixone Anchor K (Aju Medical), and Healix Knotless ${ }^{\mathrm{TM}}$ (DePuy Mitek) were used, without specific indications for use.

Immobilization after cuff repair was maintained using an abduction brace; the duration of immobilization ranged from 4 to 6 weeks, and was based on the tear size measured at the time of surgery. Shrugging of both shoulders, active elbow flexion and extension, active forearm supination and pronation, and active hand and wrist motions were encouraged immediately after surgery. Active-assisted range of motion exercises were allowed after weaning off the brace. Muscle strengthening exercises were initiated at 9 to 12 weeks postoperatively. Sports activities and heavy labor were allowed after 6 months. The rehabilitation protocol was home-based and remained constant during the study period.

\section{Outcome Evaluation}

For the radiologic outcome, perianchor cyst formation and other anchor-related radiologic changes were assessed in postoperative MRIs. Each MRI scan was obtained on a 3.0-T system (Signa HDx; GE Healthcare, Chicago, IL, USA) with a dedicated shoulder coil. The following MRI sequences were used: axial images obtained with T1-weighted spin echo sequences (repetition time [TR]/echo time [TE], 550-733/15-17 ms), and coronal and sagittal images obtained with T2-weighted spin echo sequences (TR/TE, 3,500-4,000/60-110 ms). The slice thickness was 4 $\mathrm{mm}$, with an interslice gap of 0 or $0.4 \mathrm{~mm}$, and a field of view of $16 \times 16 \mathrm{~cm}$. Perianchor cyst formation was graded in T2-weighted coronal MRI scan according to the classification of Kim et al. ${ }^{23)}$ : grade $0(\mathrm{G} 0)$, no fluid collection around the anchor; grade 1 (G1), linear fluid collection around the anchor; grade 2 (G2), local collection of fluid around any location of the anchor; grade 3 (G3), fluid collection around the entire length of the anchor and a cyst diameter less than twice the anchor diameter; and grade 4 (G4), cyst formation with a diameter larger than that in grade 3 . In patients with 2 or more anchors, the anchor with the worst grade was used. In addition, any anchor-related radiologic changes, such as signs of bone substitution or anchor displacement compared with the initial anchor position, were assessed using both MRI and X-ray images.

For the anatomical outcome, the integrity of the repaired rotator cuff was verified using MRI at least 6 months after surgery (7.8 \pm 1.2 months in the Healix group, and $6.3 \pm 0.9$ months in the Fixone group) for all patients. A postoperative period of 6 months is known to be appropriate for assessing secure tendon healing. ${ }^{21,24)}$ An experienced musculoskeletal radiologist with over 10 years of experience, who was unaware of the present study, interpreted the MRI findings and evaluated the healing of the rotator cuff to the greater tuberosity.

Functional outcomes were evaluated by the pain visual analogue scale (VAS) score and American Shoulder and Elbow Surgeons (ASES) score for all patients. The pain VAS score was measured at 3 months, 6 months and final follow-up visit (at least 1 year postoperatively), and the ASES score was assessed at least 1 year after surgery $(14.9 \pm 6.4$ months in the Healix group, and $13.2 \pm 2.1$ months in the Fixone group). The pain VAS was scored on a scale of 0 to 10, with 10 indicating the highest level of pain, and the ASES score consisted of a summation score using a 100-point system (50 points for daily function and 50 points for pain).

In addition, any complications related to the biocomposite medial anchors, such as anchor failure during the anchor insertion to the bone, deep infection, exuberant non-septic bursitis or synovitis after surgery, extensive osteolysis, or systemic allergic responses, were recorded.

\section{Statistical Analysis}

The mean values were compared using the Student's t-test

Table 2. Changes in the Functional Outcomes after Surgery

\begin{tabular}{|c|c|c|c|}
\hline Variable & Preoperative & Postoperative ${ }^{\star}$ & $p$-value \\
\hline Pain VAS score & $6.1 \pm 2.1$ & $1.2 \pm 1.2$ & $<0.001$ \\
\hline ASES score & $56.6 \pm 17.1$ & $91.1 \pm 9.9$ & $<0.001$ \\
\hline
\end{tabular}


for the continuous variables and the $\chi^{2}$ test or Fisher's exact test for the categorical variables, to determine the differences based on the kind of medial anchor used. The software IBM SPSS ver. 23.0 (IBM Co., Armonk, NY, USA) was used for all statistical analyses, and $p<0.05$ was considered statistically significant.

\section{Results}

\section{Functional Outcomes}

All functional outcomes significantly improved after surgery using the 30\% $\beta$-TCP with 70\% PLGA biocomposite suture anchors (Table 2). There were no significant differences in the ASES score between the groups. In addition, both groups showed similar pain VAS scores at every evaluation (Fig. 2).

\section{Radiologic Outcomes: Perianchor Cyst Formation}

The overall incidence of perianchor cyst formation was 23/38

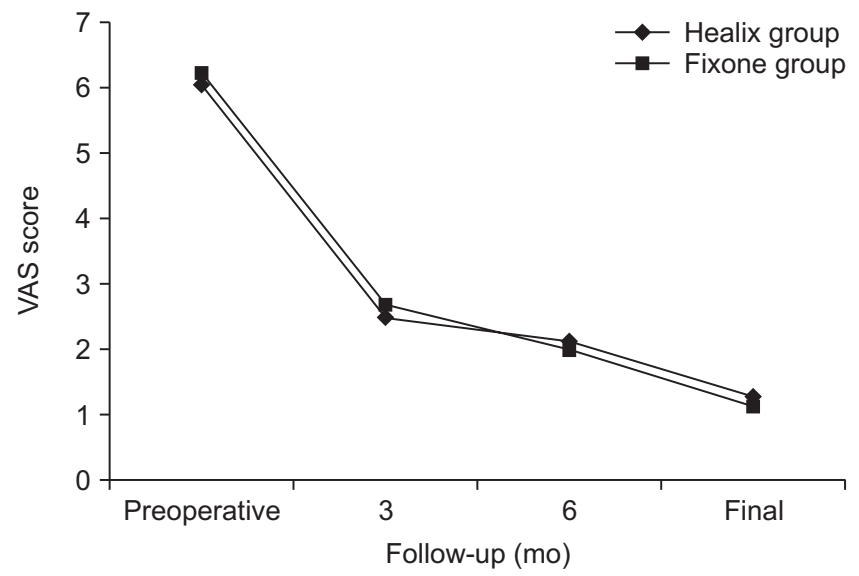

Fig. 2. Comparison of pain visual analogue scale (VAS) scores after rotator cuff repair. The Healix group includes patients who underwent arthroscopic rotator cuff repair using the Healix medial anchor (DePuy Mitek, Raynham, MA, USA), and the Fixone group includes patients who underwent the surgery using the Fixone medial anchor (Aju Medical, Seoul, Korea).
$(60.5 \%)$ in the Healix group, and $24 / 40(60.0 \%)$ in the Fixone group; no significant difference was observed in the incidence assessed at 6 months postoperatively between the groups ( $p=0.967)$ (Fig. 3). Fig. 4 exemplifies a typical case without perianchor cyst formation (grade 0 ) and one with severe perianchor cyst formation (grade 4). There was no sign of anchor absorption or bone substitution, and late anchor displacement or pull-out, in any patient of both groups.

\section{Anatomical Outcome}

Healing failure of the repaired tendon was seen in 5 patients $(13.2 \%)$ in the Healix group and 6 patients $(15.0 \%)$ in the Fixone group, with no significant differences between the groups $(p=0.815)$. In both groups, each healing failure occurred the lateral tendon-to-bone repair site, without remnant cuff tissues at the insertion site (type 1 retear ${ }^{1)}$ according to the classification

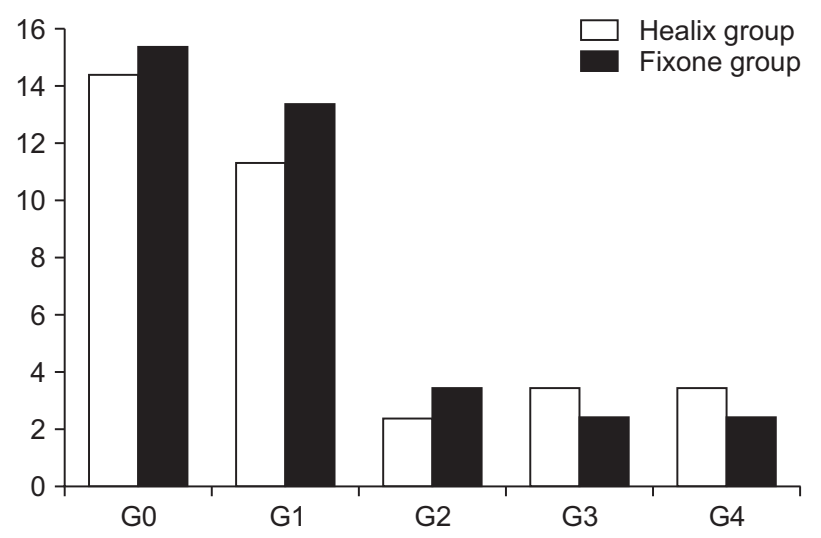

Fig. 3. Distribution of the grade of perianchor cyst formation between the groups. Perianchor cyst formation is graded on T2-weighted coronal magnetic resonance imaging scan according to the classification of Kim et al. ${ }^{23)}$ G: grade, G0: no fluid collection around the anchor, G1: linear fluid collection around the anchor, G2: local collection of fluid around any location of the anchor, G3: fluid collection around the entire length of the anchor and a cyst diameter less than twice the anchor diameter, G4: cyst diameter larger than that in grade 3 .
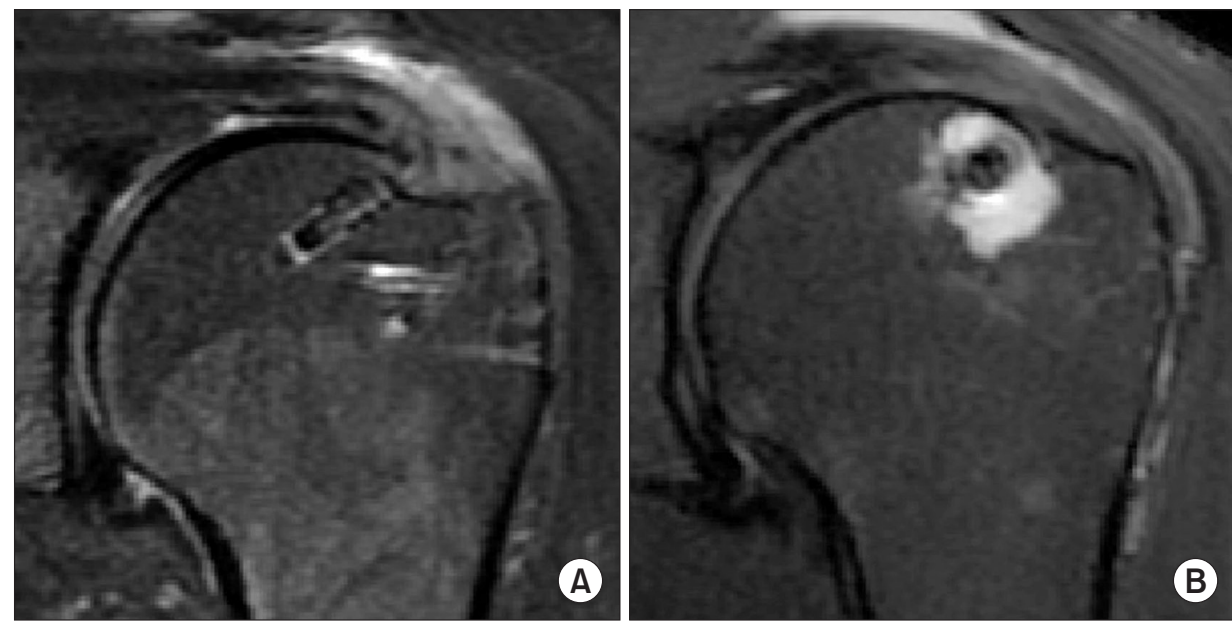

Fig. 4. Typical cases illustrating (A) without perianchor cyst formation (grade 0 ), and (B) with severe perianchor cyst formation (grade 4). 
Table 3. Comparison of the Outcomes after Rotator Cuff Repair between the Groups

\begin{tabular}{lccc}
\multicolumn{1}{c}{ Variable } & Healix group $(\mathrm{n}=38)$ & Fixone group $(\mathrm{n}=40)$ & $p$-value \\
\hline Pain VAS score at the final follow-up & $1.3 \pm 1.1$ & $1.2 \pm 1.3$ & 0.727 \\
ASES score at the final follow-up & $91.1 \pm 6.8$ & $91.2 \pm 11.2$ & 0.969 \\
Re-tear rate & $5(13.2)$ & $6(15.0)$ & 0.815 \\
Perianchor cyst formation (G0:1:2:3:4) & $15: 12: 3: 4: 4$ & $16: 14: 4: 3: 3$ & 0.967 \\
\hline
\end{tabular}

Values are presented as mean \pm standard deviation, number (\%), or number only. The Healix group includes patients who underwent arthroscopic rotator cuff repair using the Healix medial anchor (DePuy Mitek, Raynham, MA, USA), and the Fixone group includes patients who underwent the surgery using the Fixone medial anchor (Aju Medical, Seoul, Korea). Perianchor cyst formation is graded in accordance with the classification of Kim et al. ${ }^{23}$

VAS: visual analogue scale, ASES: American Shoulder and Elbow Surgeons, G: grade.

of Cho et al. $\left.{ }^{25}\right)$. The radiologic, anatomical and functional outcomes are summarized in Table 3.

\section{Complications}

Overall, 2 Healix anchors and 3 Fixone anchors were broken at the anchor body during anchor insertion to the bone. One anchor from the Fixone group was so strongly secure, that it could not be pulled out after breakage. Hence, instead of removing it, we resorted to grinding the protruding portion of the anchor using a burr. The other 4 anchors were replaced with new anchors after additionally tapping the hard bone (all patients with anchor breakage showed excellent bone qualities where the anchor was inserted) using a threaded tapper instead of a non-threaded punch. There were no cases of deep infection, exuberant nonseptic bursitis or synovitis, severe osteolysis around the medial anchor, or systemic allergic responses in either group.

\section{Discussion}

In this study, we obtained similar clinical results for perianchor cyst formation incidence and anatomical and functional outcomes after arthroscopic rotator cuff repair of medium-tolarge-sized tears, using the Fixone anchor $\mathrm{B}$ and the Healix $\mathrm{BR}^{\mathrm{TM}}$ anchor. This seems to be a logical outcome, considering that components of the Fixone anchor (30\% $\beta$-TCP and 70\% PLGA) and the Healix anchor are similar. Moreover, there was no significant complication that affected the outcome in either group.

There were few incidences of anchor-related complications for these biocomposite suture anchors, which were not severe in nature. Previously, the early-stage bioabsorbable suture anchors were reported to cause severe anchor-related complications such as extensive osteolysis ${ }^{26)}$ severe inflammatory bursitis, ${ }^{27)}$ late anchor dislodgment, ${ }^{28)}$ premature degradation, ${ }^{10)}$ or systemic allergic response." However, the biocomposite suture anchors used in this study, comprising 30\% $\beta$-TCP and 70\% PLGA, did not result in any severe complications, although there were several occurrences of minor complications such as anchor breakage, which could be solved immediately during surgery. The major reason for the reported complications of the bioabsorbable suture anchors seems to be caused by the rapid degradation rate and low strength characteristics of PGA present in the bioabsorbable anchor. ${ }^{10)}$ With the use of copolymers such as PLDLA or PLGA, most major complications seem to be resolved. Nevertheless, we observed that the problems of anchor breakage (5/78, $6.4 \%$ ) still existed in the $30 \% \beta$-TCP and $70 \%$ PLGA copolymer biocomposite anchor used in this study. Anchor breakage in all 5 patients occurred when we attempted to insert the anchor into a small punched hole against a hard bone with force. Hence, a careful approach for patients with hard bones may be needed to avoid such breakages. We recommend the use of a threaded tapper instead of a non-threaded punch for the hard bones.

In this study, the incidence of perianchor cyst was similar for both the Healix and the Fixone groups $(p=0.967)$; however, the incidence was much higher in this study $(60.3 \%)$ than in the previous study of Kim et al. ${ }^{23)}$ who showed only $7.9 \%$ of incidence for $\beta$-TCP/PLGA biocomposite suture anchors. In addition, Barber et al. ${ }^{29)}$ recently reported in their systematic review paper that the pooled incidence of cyst formation for $\beta$-TCP/PLGA biocomposite suture anchors was $4 \%$. We think that this variation may have resulted from the difference in the MRI evaluation period. Kim et al. ${ }^{23)}$ evaluated the perianchor cyst formation on MRI at a relatively later postoperative period of $12.6 \pm 1.8$ months as compared to our study ( $7.0 \pm 1.3$ months). In addition, the image evaluation time of the review article of Barber et al. ${ }^{29)}$ was much later, with a median 30 months follow-up. In a late postoperative period, the anchor inserted into the bone and the foreign body reaction because of the anchors may be more stabilized, and perianchor cyst formation may be consequently reduced. In this study, most of the perianchor cyst detected at a mean of 7 months after surgery was grade 1 perianchor cyst (26/47 [55.3\%] perianchor cyst), and we believe this lower grade perianchor cyst would disappear with time in accordance with the graft stabilization, resorption and bony ingrowth. Furthermore, the difference of raters between studies or any measurement errors may also affect the measurement results. The evaluation of inter- and intra-rater reliabilities for the detection and grading of perianchor cyst formation is therefore required. In addition, no anchor in this study was absorbed or replaced by bones at approximately 
7 months after surgery. This was also probably due to the early evaluation period, considering that PLGA copolymers are known to be absorbed between 18 and 24 months, and replaced by bone by 24 months after surgery. ${ }^{30)}$ The evaluation of perianchor cyst formation using 30\% $\beta$-TCP and 70\% PLGA biocomposite anchor in a longer follow-up period, compared with that of other anchors with different components at various insertion points, would be the next step of our study.

The current study has several limitations. First, there is the inherent limitation of this being a retrospective study, and not all patients who underwent arthroscopic repair of rotator cuff tear were included, owing to the strict inclusion criteria. This dropout from the cohort might have resulted in the risk of selection bias of which we were not aware. Second, because a single surgeon performed the surgery over a period of time, improvement of surgical technique may have affected the outcome. Third, the postoperative MRI findings were checked at a relatively early postoperative period of approximately 7 months after surgery. A long-term history or course of perianchor cyst was not confirmed in this study. In addition, the MRI examination period was not exactly similar between the two groups $(7.8 \pm 1.2$ months in the Healix group, and $6.3 \pm 0.9$ months in the Fixone group), which may cause a subtle difference in the incidence and degree of perianchor cyst formation. Further studies with MRI evaluations and longer follow-up periods are therefore necessary. Fourth, we did not evaluate differences in the use of lateral row anchors between groups. The types and compositions of the lateral row anchors may affect outcomes differently or in relation to the medial row anchors. This could therefore be a topic of further study. Finally, we compared anchors with different designs (Healix anchor, dual thread design; Fixone anchor, uniform thread design) and different size (Healix anchor, 4.5-mm; Fixone anchor, 5.5-mm). These differences in the design and size of anchors may affect the mechanical properties of anchors such as early micromotion and further perianchor cyst formation. In addition, since the differing anchor size indicates a different anchor mass, this may lead to a variation in the perianchor cyst formation, and in anchor resorption and bone substitution time. Further studies with the same design and size of anchors, as well as the material composition, are required.

\section{Conclusion}

In conclusion, no differences were observed in any of the clinical outcomes between the Healix and Fixone groups, with no major anchor-related complications.

\section{References}

1. Fehringer EV, Sun J, VanOeveren LS, Keller BK, Matsen FA 3rd. Full-thickness rotator cuff tear prevalence and correlation with function and co-morbidities in patients sixty-five years and older. J Shoulder Elbow Surg. 2008;17(6):881-5.

2. Silver MD, Daigneault JP. Symptomatic interarticular migration of glenoid suture anchors. Arthroscopy. 2000;16(1):102-5.

3. Gaenslen ES, Satterlee CC, Hinson GW. Magnetic resonance imaging for evaluation of failed repairs of the rotator cuff. Relationship to operative findings. J Bone Joint Surg Am. 1996;78(9):1391-6.

4. Benson EC, MacDermid JC, Drosdowech DS, Athwal GS. The incidence of early metallic suture anchor pullout after arthroscopic rotator cuff repair. Arthroscopy. 2010;26(3):310-5.

5. Barber FA, Snyder SJ, Abrams JS, Fanelli GC, Savoie FH 3rd. Arthroscopic Bankart reconstruction with a bioabsorbable anchor. J Shoulder Elbow Surg. 2003;12(6):535-8.

6. Athwal GS, Shridharani SM, O'Driscoll SW. Osteolysis and arthropathy of the shoulder after use of bioabsorbable knotless suture anchors. A report of four cases. J Bone Joint Surg Am. 2006;88(8):1840-5.

7. Freehill MQ, Harms DJ, Huber SM, Atlihan D, Buss DD. PolyL-lactic acid tack synovitis after arthroscopic stabilization of the shoulder. Am J Sports Med. 2003;31(5):643-7.

8. Kelly JD 2nd. Disintegration of an absorbable rotator cuff anchor six weeks after implantation. Arthroscopy. 2005;21(4):495-7.

9. Mastrokalos DS, Paessler HH. Allergic reaction to biodegradable interference poly-L-lactic acid screws after anterior cruciate ligament reconstruction with bone-patellar tendon-bone graft. Arthroscopy. 2008;24(6):732-3.

10. Speer KP, Warren RF, Pagnani M, Warner JJ. An arthroscopic technique for anterior stabilization of the shoulder with a bioabsorbable tack. J Bone Joint Surg Am. 1996;78(12):1801-7.

11. Nusselt $\mathrm{T}$, Freche $\mathrm{S}$, Klinger HM, Baums MH. Intraosseous foreign body granuloma in rotator cuff repair with bioabsorbable suture anchor. Arch Orthop Trauma Surg. 2010;130(8):103740.

12. Suchenski M, McCarthy MB, Chowaniec D, et al. Material properties and composition of soft-tissue fixation. Arthroscopy. 2010;26(6):821-31.

13. Nho SJ, Provencher MT, Seroyer ST, Romeo AA. Bioabsorbable anchors in glenohumeral shoulder surgery. Arthroscopy. 2009;25(7):788-93.

14. Barber FA, Dockery WD, Cowden CH 3rd. The degradation outcome of biocomposite suture anchors made from poly Llactide-co-glycolide and $\beta$-tricalcium phosphate. Arthroscopy. 2013;29(11):1834-9.

15. Kim SH, Kim DY, Kwon JE, Park JS, Oh JH. Perianchor cyst formation around biocomposite biodegradable suture anchors after rotator cuff repair. Am J Sports Med. 2015;43(12):290712.

16. Randelli P, Compagnoni R, Aliprandi A, et al. Long-term degradation of poly-lactic co-glycolide/ $\beta$-tricalcium phosphate 
biocomposite anchors in arthroscopic bankart repair: a prospective study. Arthroscopy. 2014;30(2):165-71.

17. Yang IC, Kim SY, Kang HC, Lee HY, Kwon JY, Kim SM. A study on evaluation of bioabsorbable anchor (PLGA $+\beta-\mathrm{TCP})$ through mechanical test under moisture difference. J Med Devices. 2016;10(3):1-2.

18. Chung SW, Kim JY, Kim MH, Kim SH, Oh JH. Arthroscopic repair of massive rotator cuff tears: outcome and analysis of factors associated with healing failure or poor postoperative function. Am J Sports Med. 2013;41(7):1674-83.

19. Aziz SR. The division of oral and maxillofacial surgery at columbia presbyterian medical center: a.k.a. HSDM South. Harv Dent Bull. 1999;8(1):26-8.

20. DeOrio JK, Cofield RH. Results of a second attempt at surgical repair of a failed initial rotator-cuff repair. J Bone Joint Surg Am. 1984;66(4):563-7.

21. Goutallier D, Postel JM, Bernageau J, Lavau L, Voisin MC. Fatty muscle degeneration in cuff ruptures. Pre- and postoperative evaluation by CT scan. Clin Orthop Relat Res. 1994;(304):7883.

22. Fuchs B, Weishaupt D, Zanetti M, Hodler J, Gerber C. Fatty degeneration of the muscles of the rotator cuff: assessment by computed tomography versus magnetic resonance imaging. J Shoulder Elbow Surg. 1999;8(6):599-605.

23. Kim SH, Oh JH, Lee OS, Lee HR, Hargens AR. Postoperative imaging of bioabsorbable anchors in rotator cuff repair. Am J
Sports Med. 2014;42(3):552-7.

24. Uhthoff HK, Seki M, Backman DS, Trudel G, Himori K, Sano H. Tensile strength of the supraspinatus after reimplantation into a bony trough: an experimental study in rabbits. J Shoulder Elbow Surg. 2002;11(5):504-9.

25. Cho NS, Yi JW, Lee BG, Rhee YG. Retear patterns after arthroscopic rotator cuff repair: single-row versus suture bridge technique. Am J Sports Med. 2010;38(4):664-71.

26. Glueck D, Wilson TC, Johnson DL. Extensive osteolysis after rotator cuff repair with a bioabsorbable suture anchor: a case report. Am J Sports Med. 2005;33(5):742-4.

27. Sivaloganathan S, Amr R, Shrivastava R, Relwani J. The Risotto sign - a severe inflammatory bursitis with rice body formation, complicating a rotator cuff repair with a bioabsorbable suture anchor. JRSM Open. 2015;6(1):2054270414562986.

28. Magee T, Shapiro M, Hewell G, Williams D. Complications of rotator cuff surgery in which bioabsorbable anchors are used. AJR Am J Roentgenol. 2003;181(5):1227-31.

29. Barber FA, Spenciner DB, Bhattacharyya S, Miller LE. Biocomposite implants composed of poly(Lactide-co-Glycolide)/ $\beta$-tricalcium phosphate: systematic review of imaging, complication, and performance outcomes. Arthroscopy. 2017;33(3):683-9.

30. Kurtz SM, Devine JN. PEEK biomaterials in trauma, orthopedic, and spinal implants. Biomaterials. 2007;28(32):4845-69. 